Public Abstract

First Name:Dario

Middle Name:Omar

Last Name:Cersosimo

Adviser's First Name:Craig

Adviser's Last Name:Kluever

Co-Adviser's First Name:Douglas

Co-Adviser's Last Name:Smith

Graduation Term:SP 2011

Department:Mechanical \& Aerospace Engineering

Degree:PhD

\title{
Title:EVALUATION OF NOVEL HOVERING STRATEGIES TO IMPROVE GRAVITY-TRACTOR DEFLECTION MERITS
}

The gravity-tractor (GT) consists of a spacecraft hovering inertially over a small asteroid. This equilibrium state is achieved by the action of a pair of engines that balance the gravitational acceleration. Due to Newton's law of gravitation the spacecraft causes a small gravitational pull on the asteroid that after prolonged time intervals causes a small change in its trajectory preventing it from impacting the Earth. This dissertation introduces a novel concept in the implementation of the GT to augment its deflection merits.

Two novel guidance laws are designed to take advantage of the asteroid shape and rotation rate forcing the GT spacecraft to move towards and away the center of mass of the asteroid in synchronous motion with its rotation, resulting in an increased gravitational pull. The asteroid model was generalized as a solid, homogeneous triaxial ellipsoid. A small GT spacecraft was proposed and a detailed model of the NSTAR ion thrusters was used to investigate the efficiency of the GT operating under these novel guidance laws and the classical inertial hovering. The performance of these hovering laws was examined over a wide range of asteroid shapes and rotation rates. The results obtained suggest that it is possible to improve the deflection merits by up to $60 \%$. The propellant efficiency showed to be sensitive to the spacecraft parameters; in particular, the engine model and the controller used to sustain the desired hovering state play an important role in the propellant penalties associated with these extended hovering modes. Furthermore, these results indicate that previous works have overestimated the propellant efficiency of a typical GT by using oversimplified models of solar electric engines. 